\title{
Lauric Arginate/Cellulose Nanocrystal Nanorods- Stabilized Alkenyl Succinic Anhydride Pickering Emulsion: Enhancement of Stabilization and Paper Sizing Performance
}

\section{Yucheng Li}

Qilu University of Technology

Dehai Yu ( $\square$ yudehai@qlu.edu.cn )

Qilu University of Technology https://orcid.org/0000-0003-4286-3616

\section{Xiangyu Wang}

Qilu University of Technology

\section{Qiang Wang}

Qilu University of Technology

\section{Zhen Zhang}

South China Normal University

Wenxia Liu

Qilu University of Technology

\section{Research Article}

Keywords: Lauric arginate, Cellulose nanocrystal, Interfacial adsorption, Pickering emulsions, Paper sizing

Posted Date: December 23rd, 2021

DOI: https://doi.org/10.21203/rs.3.rs-1126328/v1

License: (c) (i) This work is licensed under a Creative Commons Attribution 4.0 International License. Read Full License

Version of Record: A version of this preprint was published at Cellulose on March 14th, 2022. See the published version at https://doi.org/10.1007/s10570-022-04502-z. 


\section{Abstract}

Biodegradable colloidal particle materials are becoming attractive candidates as eco-friendly chemical additives in the low-carbon economy era. However, developing cheap, stable, and efficient paper-sizing agents is still a challenging issue both for paper making academic community and industry. Here, an easy-fabricating, stable, and high-performance alkenyl succinic anhydride (ASA) paper-sizing emulsion is developed, which is stabilized by lauric arginate (LAE)/cellulose nanocrystal (CNC) nanorods. Furthermore, the chemical crosslinking between ASA and LAE/CNC nanorods can adjust the wettability and interfacial adsorption of $L A E / C N C$, resulting in improved stability of ASA-sizing emulsion. This novel paper-sizing emulsion shows a small droplet size $(0.8 \mu \mathrm{m})$, high hydrolysis resistance, and a high papersizing degree (300 s) along with a remarkable hydrophobicity contact angle of $110^{\circ}$ for long-term storage. This work opens a door for realizing an interfacial self-assembled Pickering-stabilizer, which leads to an environmentally friendly, pervasive and cost-effective emulsification technique for the next-generation paper-sizing additives.

\section{Introduction}

Emulsion is vital in the production of food, medicine, paper and cosmetics industries, owing to its specific physicochemical and biological properties, such as excellent stability and resistance to liquid penetration. However, the traditional emulsion has disadvantages in terms of long-term stability. Therefore, solid particles are applied to stabilize the emulsion and named as Pickering emulsion, in which they are adsorbed on the oil-water interface, and form a mechanical barrier with densely packed particles (Pickering, 1907). In particular, Pickering emulsions stabilized by biodegradable colloidal particles are attracting increasing interest, since the demand for merchandise made of environment-friendly ingredients is growing (Dickinson, 2017; McClements \& Gumus, 2016). Therefore, many types of proteins and/or polysaccharide-based colloidal particles have been developed as environment-friendly Pickering emulsifiers (Hong et al., 2018; Sun et al., 2021). Pickering emulsion shows better stability than traditional surfactant-stabilized emulsion due to the irreversible adsorption of particles even at a low particle concentration (Xia et al., 2018).

It is well-known that sizing agent is an important functional additive imparting paper with resistance to liquid penetration. Among them, alkenyl succinic anhydride (ASA) is a commonly applied neutral sizing agent, and needs to be prepared as oil in water emulsion before sizing (G. Li, Song, Liu, Yu, \& Wang, 2017; Yu et al., 2020). In the traditional emulsifying process, excessive surfactants can facilitate emulsification, but it will have an adverse effect on sizing (Yu, Lin, \& Li, 2013). In addition, ASA is easily hydrolyzed, the hydrolysate will have obstacles on sizing effect and paper-making process (Mohit, Bhadra, Goswami, \& Agarwal, 2007; Robert, 1990). Based on problems mentioned above, the preparation and application of ASA emulsion are complex and difficult to control, which cause unstable sizing effect (Hubbe, 2007). In practical production, ASA requires on-site emulsification preparation and immediate usage. In the meantime, nano-clay and the modified particles-stabilized ASA emulsion exhibit good performance and are expect to solve the current issues (Yucheng Li et al., 2021; Yu et al., 2020). However, clays and their 
modified products are not degradable and prone to high viscosity of the emulsion through forming gel system in continuous phase (Wang, Deng, Sun, \& Yang, 2020; Y. Zhang et al., 2021). Therefore, it is necessary to explore more efficient and environmentally friendly solid emulsifier, and provide reliable methods for green paper additives. Furthrmore, the Pickering emulsion system is expected to provide a potential alternative method for paper sizing emulsion, for large-scale manufacturing of paper companies and the rapid development of paper machines (Bai et al., 2021).

Cellulose nanocrystal (CNC) is an effective Pickering stabilizer for oil-in-water (O/W) emulsion due to its superior properties in terms of reusability, sustainability, non-toxicity and biocompatibility (Chen, Liu, \& Tang, 2019; Chu, Sun, Wu, \& Xiao, 2020; Habibi, Lucia, \& Rojas, 2010; Kedzior, Gabriel, Dubé, \& Cranston, 2020). CNC can adsorb at the water-oil interface and form a dense interface network, which is derived from its wettability, and rod-like nanostructure (Capron, Rojas, \& Bordes, 2017; Lu et al., 2020). Nonetheless, the high hydrophilicity of CNC limits its application in Pickering emulsions, and CNC is generally modified to improve its emulsification performance(Du, Guo, Li, \& Gao, 2017; Patel, Lakshmibalasubramaniam, Nayak, \& Camire, 2021; Saidane, Perrin, Cherhal, Guellec, \& Capron, 2016). Chemical modification of CNC is a widely used strategy(Cunha, Mougel, Cathala, Berglund, \& Capron, 2014; Lu et al., 2020; Tang et al., 2014; Zoppe, Venditti, \& Rojas, 2012), but physical adsorption with surfactant or macromolecule is a more promising strategy(Ben Azouz, Ramires, Van den Fonteyne, El Kissi, \& Dufresne, 2012; Gong, Wang, \& Chen, 2017; Huang, Ye, \& Berry, 2016; Patel et al., 2021; Tardy et al., 2017) because of its non-toxicity, biocompatibility, and easy processing. Since CNC is negatively charged, cationic organic polymer can bind with CNC through electrostatic adsorption. Lauric arginate (LAE) is a biological and amino acid-based cationic surfactant(Bai, Xiang, Huan, \& Rojas, 2018), which is synthesized from lauric acid, arginine and ethanol. It can adsorb onto the surface of CNC, improve their emulsification property, and retain their sustainability (Bai et al., 2018; Chi \& Catchmark, 2017). The colloid behavior of LAE/CNC complex is controllable and regulatable at the oil-water interface, which plays a very unique and important role in stabilizing the Pickering emulsion system (Yanan Li et al., 2018).

Herein, the ASA sizing Pickering emulsion stabilized with LAE-modified CNC was prepared. The emulsification mechanism was systematically studied by analyzing the cross-linkage between LAE/CNC and ASA, and the adsorption of LAE/CNC at the water-oil interface. Scheme 1 shows that ASA Pickering emulsion could be obtained by addition of LAE/CNC NRs. The composition and structure of the obtained emulsions as well as the sized paper were analyzed by scanning electron microscopy (SEM), atomic force microscopy (AFM), transmission electron microscopy (TEM), laser scanning confocal microscope (CLSM), fourier transform infrared (FTIR) spectroscopy, and X-ray nanocomputed tomography (nano-CT). Subsequently, the water-repellent and hydrolysis resistance capacity of the sized paper was evaluated. It is expected to prepare an environmentally friendly ASA papermaking sizing emulsion with enhanced sizing performance and higher stability. The introduction of functionalized cellulose nanocrystals into ASA emulsion to prepare superior sizing emulsion is a long-term project, which will enrich the colloid interface chemistry and facilitate application technology of emulsion additives for papermaking. 


\section{Materials And Methods \\ 2.1 Materials}

The cationic, food-grade surfactant ethyl- $\mathrm{N}^{\mathrm{a}}$-lauroyl-L-arginate hydrochloride ( $\geq 98 \%$ purity, $\mathrm{C}_{20} \mathrm{H}_{41} \mathrm{~N}_{4} \mathrm{O}_{3} \mathrm{Cl}, \mathrm{MW} \sim 421.02 \mathrm{~g} / \mathrm{mol}$ ) was bought from Maclin (Shanghai, China). Sulfonate cellulose nanocrystal (CNC) was a needle-like nanomaterial obtained by sulfuric acid treatment of natural plant cellulose. The surface contains sulfonic acid groups and hydroxyl groups, was purchased by ScienceK Co., Ltd. (Hu Zhou, China). ASA oil with the main ingredient of octadecenyl succinic anhydride was supplied by Kamira (Shanghai, China). Nile red and Nile blue dyes were purchased from Maclean (Shanghai, China). Aspen alkaline peroxide mechanical pulp (APMP) was provided in a pulp board form by Shandong Zhongmao Shengyuan Pulping Co., Ltd. Ultrapure water with a resistivity of $18.2 \mathrm{M} \Omega \cdot \mathrm{cm}$ was produced by a Milli-Q water system attached to an ELGA reverse osmosis unit. Other chemicals used were all analytical reagents.

\subsection{Preparation and characterization of LAE/CNC nanorods}

Concentrated LAE was diluted to $1.0 \mathrm{wt} \%$ by Milli-Q water. The CNC hydrogel was first diluted and then LAE was added to obtain the desired suspended CNC concentrate. Briefly, LAE was first added to the CNC suspension and gently shaken for $20 \mathrm{~s}$, followed by sonicating with an ultrasonic generator (YQ-620C, Shanghai Yi Jing Ultrasonic Instrument Co., Ltd., China) at $25{ }^{\circ} \mathrm{C}$ for $10 \mathrm{~min}$. The prepared nanorods were left at $25{ }^{\circ} \mathrm{C}$ before analysis.

Fourier transform infrared spectroscopy (FT-IR) spectra of LAE/CNC were measured by a FTIR spectrophotometer (Bruker Vertex V70, USA) in absorbance mode with the attenuated total reflection (ATR) diamond sensor. Spectra were collected over $600-4000 \mathrm{~cm}^{-1}$ regions at ambient conditions at a resolution of $4 \mathrm{~cm}^{-1}$. All the LAE/CNC suspension samples were filtered by a B-GLM22 syringe filter (BKMAN, China) with $0.22 \mu \mathrm{m}$ of membrane pore size before measurement. The particle size distribution and zeta potential of the samples were determined by dynamic light scattering measurement using a Nano-ZS Zetasizer (Malvern, U.K.) angle with a He-Ne laser (laser wavelength $633 \mathrm{~nm}$ ) at a $178^{\circ}$ scattering and temperature of $298 \mathrm{~K}$. To prevent multiple scattering effects, samples were diluted to a concentration of $0.005 \mathrm{wt} \%$ with $\mathrm{MQ}$ water before measuring and repeated three times. The three-phase contact angles of LAE/CNC were measured with an OCA-50 micro contact angle measuring tool (Dataphysics, Germany) using a conventional particle-platelet method (Yucheng Li et al., 2021). Before starting the test, the samples were modulated as 2 mm-thick circular pieces with an HY-12 tablet machine (Tianguang, China). The Protractor software was employed to determine the contact angle via axisymmetric drop shape analysis. The contact angle of each sample was measured three times, and the average of per sample was reported. Atomic force microscope (AFM, Bruker, USA) was employed to measure the morphology of $\mathrm{LAE} / \mathrm{CNC}$ nanorods in tapping mode. Before inspection, a $10 \mu \mathrm{L}$ drop of LAE/CNC suspension with concentration of $5 \mu \mathrm{g} / \mathrm{mL}$ was deposited onto a mica substrate $(20 \times 20 \mathrm{~mm})$ and dried at $25{ }^{\circ} \mathrm{C}$ overnight. A video optical contact angle measurement instrument (OCA-50, Data 
Physics Instruments, Germany) was used to study the surface tension and interface tension of the LAE/CNC suspension using pendant drop method. ASA oil was added to a cuvette, and the transmissivity of the oil container was checked prior to measurement. In brief, the pendant droplets of $5 \mu \mathrm{L}$ of solution, containing LAE/CNC nanorods, was formed at the end of a low retention pipet tip immersed in oil phase. The droplets were left for $10 \mathrm{~s}$ at $25^{\circ} \mathrm{C}$ before measurement. The optical turbidities of LAE/CNC suspension were characterized by UV-VIS spectrophotometer (Agilent 8453, U.S) at a wavelength of 600 nm operated at $25^{\circ} \mathrm{C}$.

\subsection{Preparation and characterization of ASA Pickering emulsions stabilized by LAE/CNC NRs}

LAE/CNC NRs-stabilized Pickering emulsions were prepared as follows: the LAE/CNC NRs suspension was prepared and placed in a plastic tube, followed by ASA oil addition to the suspension and sonication using an ultrasonic generator (YQ-620C, Shanghai Yi Jing Ultrasonic Instrument Co., Ltd., China) with an immersed titanium microtip. In this study, the oil-water ratio of LAE/CNC NRs stabilized ASA Pickering emulsions is $1: 2(\mathrm{v}: \mathrm{v})$.

The stability of emulsions with respect to creaming and coalescence was evaluated by counting the volume of discharged oil and water in $24 \mathrm{~h}$ via a FinePix S4050 digital camera (Fujifilm, Japan). The stability is expressed as emulsifying index (EI):

$\mathrm{El}=\mathrm{H}_{\mathrm{e}} / \mathrm{H}_{\mathrm{E}}$

where $H_{E}$ is the total emulsion's height and $H_{e}$ is the serum layer's height. The values of $H_{E}$ and $H_{e}$ for different samples were measured via visual analysis of the samples' photographs using ImageJ software. A DM750M optical microscope (Leica, Germany) was applied to check ASA Pickering emulsions' morphology without further dilution. The average size of droplet and its distribution in the prepared emulsions were analyzed by ImageJ. For visualizing and distinguishing LAE/CNC and ASA oil in the emulsion system, the ASA oil and LAE/CNC suspension were dyed with Nile blue and Nile red respectively before emulsion preparation. Then, the emulsion droplets were observed under a laser confocal microscope. The adsorption of particles on the surface of emulsion droplet was analyzed by confocal laser scanning microscope (CLSM, Leica, SP8, Germany). The FT-IR spectra of ASA emulsions stabilized by LAE/CNC NRs were measured using a FT-IR spectrophotometer (Vertex V70, Bruker, USA) under an absorbance mode via an attenuated total reflection (ATR) diamond sensor.

Dried ASA droplets loaded with LAE/CNC were inspected under scanning electronic microscope (SEM, Hitachi, Regulus8220, Japan) and transmission electron microscope (TEM, Hitachi Koki Co., Ltd., JEM 2100, Japan). A $10 \mu \mathrm{L}$ drop of ASA emulsions with concentration of $0.02 \mathrm{~g} / \mathrm{mL}$ was deposited onto a carbon membrane support (T11023, Beijing XXBR Technology Co., Ltd.) and dried at $25^{\circ} \mathrm{C}$ overnight before inspection. Also, the morphology of ASA emulsions was measured by atomic force microscope (AFM, Bruker, USA) under tapping mode. A $10 \mu \mathrm{L}$ drop of ASA emulsions with concentration of $0.02 \mathrm{~g} / \mathrm{mL}$ was deposited onto a mica substrate $(20 \times 20 \mathrm{~mm})$ and dried at $25^{\circ} \mathrm{C}$ overnight before inspection. The 
data of emulsions' creaming stability were logged by a Turbiscan device (LUMiSizer, LUM Jiangsu Instruments Co., Ltd., China). Dynamic changes were evaluated by laser light's backscattering intensity as a function of height during creaming. After high-speed centrifugation $\left(895 \times \mathrm{g}, 10 \mathrm{~min}\right.$ at $\left.25^{\circ} \mathrm{C}\right)$, samples were prepared in rectangle plastic tubes $(10 \times 10 \times 100 \mathrm{~mm})$. The viscosities of samples were measured by a rheometer (MCR 300, Anton Paar, Germany) using parallel plates (PP25) with the gap fixed at 1.0 $\mathrm{mm}$. The corresponding viscosity was recorded with a shear rate ranging from $0.01 \mathrm{~s}^{-1}$ to $100 \mathrm{~s}^{-1}$. The dynamic viscoelastic range was derived by strain sweep from $0.01-100 \%$ at the constant frequency of 10 $\mathrm{rad} / \mathrm{s}$. A dynamic frequency sweep was conducted at an $1.0 \%$ constant strain and wide frequency range of 0.1 to $100 \mathrm{rad} / \mathrm{s}$. All measurements were made at $25^{\circ} \mathrm{C}$.

\subsection{Handsheet making and sizing performance of ASA emulsion}

A PTI type paper former (ISO 5269-2, Austria) was used to prepare ASA-sized paper handsheets with a 60 $\mathrm{g} / \mathrm{m}^{2}$ base weight. The pulp was first soaked in water overnight and then dispersed at $20 \mathrm{~g} / \mathrm{L}$ by a standard disintegrator (Lorentzen \& Wettre, Sweden) at 20,000 rpm, followed by purification using a PFI mill (KRK Company, Japan). ASA-sized paper was prepared using a CUF5/200 laboratory coating unit (Sumet Technologies Ltd., Germany), The as-prepared ASA emulsion was diluted to $1.0 \mathrm{wt} \%$ before paper sizing, which was quantitatively determined by the liquid permeation method using GB/T460-2008 (Tan et al., 2014), and further used for quantitative determination. An automatic video optical contact angle measurment instrument (OCA-50, Dataphysics, Germany) was used to measure the static water contact angle of the sized paper at various time points. The 3D structural images of sized paper were derived by a multi-scale X-ray nanocomputed tomography (nano-CT) system (Skyscan 2211, Bruker, Germany). The size of the tested sample paper was $2 \mathrm{~mm} \times 2 \mathrm{~mm}$.

\section{Results And Discussion}

\subsection{Characterization of LAE/CNC nanorods}

The FT-IR spectra of CNC and LAE-modified CNC are displayed and compared in Fig. 1a. It is shown that two new bands at 2915 and $2851 \mathrm{~cm}^{-1}$ were found in the spectra of LAE/CNC, which were assigned to the asymmetric and symmetric stretching of $\mathrm{CH}_{2}$ in the long alkyl chain of LAE. Meanwhile, the peaks of $3420 \mathrm{~cm}^{-1}$ and $1756 \mathrm{~cm}^{-1}$ were corresponded to the vibration of $\mathrm{O}-\mathrm{H}$ and carbonyl group on side-chain from LAE. In addition, the signals at $1643 \mathrm{~cm}^{-1}$ and $1530 \mathrm{~cm}^{-1}$ were correlated with amide I (C=0 stretching) and amide II (C-N stretching and N-H deformation) from the LAE backbone respectively, which were consistent with the results of Johnson (Johnson, Zink-Sharp, \& Glasser, 2011). Overall, LAE has been successfully connected onto CNC NRs.

The surface and interface tensions of CNC nanoparticles (2.0 wt\%) with LAE solutions were derived using pendant drop method (Fig. 1b). The results showed that the surficial and interfacial tensions fall rapidly with the addition of $L A E$, and then approached to a flat until the dosage of LAE was $0.015 \%$, since the 
interaction among the hydrophobic LAE tail groups and the electrostatic interaction with CNC can induce local micellization, facilitating LAE binding to the surface of CNC nanorods. When the amount of LAE was $0.015 \sim 0.025 \mathrm{wt} \%$, surface tension kept a constant value around $45 \mathrm{mN} / \mathrm{m}$, which could be caused by the formation of free unbound micelles in the native solution, an indication of surfactant saturation binding to CNC NRs. The hydrophobicity of pristine CNC NRs and LAE/CNC NRs was measured with three-phase contact angle (Fig. 1c). It was seen that LAE/CNC NRs had an increasing contact angle to $80^{\circ}$ with the increasing dosage of LAE, and then decreased rapidly. Whereas, CNC NRs had a contact angle of $60^{\circ}$ and showed a relative hydrophilicity. So the addition of LAE increased the contact angle and hydrophobicity of CNC NRs. The LAE/CNC NRs reached the maximum contact angle about $87.6^{\circ}$ with $0.016 \% \mathrm{LAE}$, and then decreased to $36.5^{\circ}$ with $0.02 \% \mathrm{LAE}$, which could be due to the formation of hydrophilic micellar structure with excessive LAE and the reduced hydrophobicity of LAE/CNC NRs. Following LAE modification, the hydrophobicity of CNC particles increased, which may facilitate their attachment to the surface of ASA oil droplet, as well as the formation of stable oil-in-water emulsions with uniform and small spherical droplets.

To have a better understanding on the aggregation of LAE/CNC nanorods, the microstructural changes of primary $\mathrm{CNC}$ and its nanorods with different LAE concentrations can be studied by AFM imaging (Fig. 1d). It was obvious that the dispersion of CNC NRs was improved gradually with an increasing amount of $\mathrm{LAE}$, which was mainly because of the electrostatic adsorption and bridging traction action between positive charged LAE and negative charged CNC NRs. The LAE/CNC NRs showed the best dispersion and stability with $0.02 \%$ LAE. Furthermore, the LAE/CNC NRs would form bundle structures with the concentration of LAE above $0.02 \%$. The zeta potential and macro morphology of LAE/CNC NRs suspension were recorded in Fig. 1e. The absolute value of zeta potential increased firstly to $-70 \mathrm{mV}$ with $0.02 \%$ CNC NRs, and then decreased with the increasing dosage of LAE, which illustrated the optimal dispersion and stability of LAE/CNC NRs suspension with $0.02 \%$ CNC NRs. With excessive LAE (>0.02\%), the absolute value of zeta potential decreased again, which confirmed the connection between $L A E$ and CNC NRs. Otherwise, the decreased absolute value of zeta potential would result in micro-flocculation of LAE/CNC NRs, which was beneficial for the stability of Pickering emulsion (Bollhorst, Rezwan, \& Maas, 2017). Meanwhile, this variation tendency was further confirmed by the result of size distribution (Fig. 1f), and the size distribution curve of LAE/CNC NRs was the narrowest with $0.02 \%$ LAE.

\subsection{ASA Pickering emulsion stabilized by LAE/CNC NRs.}

Fig. 2a shows the CLSM image of ASA emulsion with LAE/CNC NRs. The emulsion droplets with uniform diameter were clearly observed, and their diameter had a decreasing tendency with increasing dosage of CNC NRs. The corresponding data of size distribution were analyzed from Fig. 2b; the emulsion droplets had an average diameter of $5.3 \mu \mathrm{m}$ with $0.2 \% \mathrm{CNC}$, while it was reduced to $0.8 \mu \mathrm{m}$ at $2.0 \% \mathrm{CNC}$ with a narrow size distribution curve. All freshly prepared ASA emulsion had a homogeneous appearance. Their stabilities were observed within $24 \mathrm{~h}$ and the El values were recorded. It was shown in Fig. 2c that emulsion's stability was improved by the increasing dosage of CNC, and the emulsion still kept homogeneous appearance with $2.0 \% \mathrm{CNC}$ after $24 \mathrm{~h}$. The ASA emulsion stabilized by $0.2 \%, 0.6 \%$ and 
$1.0 \%$ CNC all had separated phase and decreased El values of $92 \%, 85 \%$, and $64 \%$, respectively. Consistently, the emulsion showed a similar tendency with centrifugal stability. The microphotographs of ASA emulsion are listed in Fig. S1, and all samples were recorded with good homogeneity in $3 \mathrm{~h}$. The emulsion stabilized by low CNC concentration $(<1.8 \%)$ showed an uneven distribution of emulsion droplets, and ungraded droplets were also found in the images, which may be the agglomerated emulsion droplets caused by the incomplete coverage. With a higher dosage of CNC at $1.8 \%$ and $2.0 \%$, the emulsion droplets showed a decreased diameter and a more uniform distribution. However, the agglomerated emulsion droplets and their uneven distribution were appeared again with excessive CNC (> $2.0 \%$ ). Therefore, LAE/CNC NRs have improved emulsifying capacity and can adjust the size of Pickering emulsion.

\subsection{Effect of $\mathrm{pH}$}

The $\mathrm{pH}$ value of system is an important parameter for the stability of nanocellulose-stabilized Pickering emulsion (Kedzior et al., 2020). It was analyzed from Fig. 3a that $\mathrm{pH}$ value had influences on the size distribution and morphology of ASA emulsion. Especially at the $\mathrm{pH} \sim 7$, the emulsion has large droplets and size distribution. However, the emulsion had small droplets and good homogeneity at other $\mathrm{pH}$ values. The appearance of the LAE/CNC-stabilized ASA emulsion under different $\mathrm{pH}$ values is shown in Fig. $3 b$. The emulsification index of the freshly prepared emulsion is $100 \%$, and there is no phase precipitation. After being left for $24 \mathrm{~h}$, a large amount of turbid water phases were precipitated in the emulsion at $\mathrm{pH}>7$. This may be due to the precipitation of phases caused by the polymerization of emulsion droplets, which is consistent with the results in Fig. 3a. Given that the carbonyl lactone ring groups of ASA will be in the form of $-\mathrm{COOH}$ at a high $\mathrm{pH}$ environment, it leads to a hydrolysis reaction and reduces the stability of the emulsion (see Fig. 3c). Furthermore, when the $\mathrm{pH}>7$, hydroxy of LAE/CNC NRs interacted with carboxyl from ASA-acid at the o/w interface, leading to the oriented movement of the weakly hydrogen-bonded water molecules in the interfacial region, which could hinder the penetration of water into an oil phase and/or ASA molecular transport (Scatena, Brown, \& Richmond, 2001).

The effect of $\mathrm{pH}$ ranging from 3 to 11 on the droplet size distribution of the Pickering emulsions have given in Fig. 3a and 3c. The ASA emulsion stabilized by LAE/CNC under different pH values for $24 \mathrm{~h}$, there is almost no phase precipitation (Fig. 3b). It was analyzed that $\mathrm{H}^{+}$would reduce the repulsion between LAE/CNC NRs within acid circumstance (Patel et al., 2021). Hence, microgels were formed to resist the flocculation of emulsion. The droplet size distribution and optical micrographs of emulsions with different $\mathrm{pH}$ values after $24 \mathrm{~h}$ are listed in Fig. S3 and S4. Similar trends can be seen in Fig. 3c and $\mathrm{S} 4$ that the average droplet size of the prepared emulsion is steady except at $\mathrm{pH}$, which implies that the weakly alkaline condition is not conducive to the stability of the LAE/CNC-stabilized ASA emulsion.

\subsection{Rheological behavior of the ASA Pickering emulsions stabilized by LAE/CNC NRs}

To better understand the gel or liquid properties of as-prepared ASA Pickering emulsions, the storage modulus $(G)$ /loss modulus $\left(G^{\prime \prime}\right)$ and the viscosity with different $C_{C N C}$ and $\mathrm{pH}$ values were investigated 
(Fig. 3e-h). The result shows that the value of $G^{\prime}$ was higher than that of $G^{\prime \prime}$ in the whole range of angular frequency when the $C_{C N C}$ was above1.8\%, indicating that the emulsions' linear rheological behavior was mainly viscoelastic and the existence of well-developed elastic networks formed by the dispersed droplets in the emulsion system (Qiao, Wang, Shao, Sun, \& Miller, 2015). The enlarged difference between $G^{\prime}$ and $G^{\prime \prime}$ indicated the high resistance to flocculation (Zhang, Gu, Liu, \& Wang, 2021), especially when the $C_{C N C}$ was $2.0 \%$. Moreover, the ASA emulsion stabilized by LAE/CNC NRs with $C_{C N C} \sim 2.0 \%$ showed the maximum values of $G^{\prime}$ and $G^{\prime \prime}$, which indicates the high resistance to flocculation and explains the better stability. Setting the $\mathrm{pH}$ value of emulsion as variable, the value of $G^{\prime}$ was higher than that of $G^{\prime \prime}$ when $\mathrm{pH}$ value was 3 9 in the whole angular frequency range. Elasticity has dominated the rheological behavior of the emulsions stabilized by LAE/CNC NRs. It is worth to note that the as-prepared emulsion showed greater $G^{\prime}$ and $G^{\prime \prime}$ values at $\mathrm{pH} \sim 5$ than those at other $\mathrm{pH}$ values, suggesting that the LAE/CNC NRsstabilized emulsion at $\mathrm{pH} 5$ shows strong resistant to flocculation (Binks, Muijlwijk, Koman, \& Poortinga, 2017).

Fig. $3 g$ shows the relationship between the apparent viscosity of ASA emulsion and shear rate. All emulsions have a reduced viscosity and then tend to a constant with increasing shear rate, so emulsion showed a shearing-thinning behavior, which was due to the deformation of emulsion droplets and their ordered arrangement as well as the continuous phase (Thaiphanit, Schleining, \& Anprung, 2016). The results showed that the $C_{C N C}$ obviously influenced the rheological apparent viscosity behavior of the emulsion. Since the emulsion system's network was mostly destroyed by the shearing force, the repulsive forces were predominant at the high shear rate (Niu et al., 2018). In addition, it can be found that when the shear rate is low, the apparent viscosity of the emulsion decreases at the fastest rate. As the shear rate increases, the range of viscosity reduced is gently decreased. Therefore, the ASA Pickering emulsion prepared by using modified CNC as an emulsifier has the typical characteristics of pseudoplastic fluid. The concentration of CNC affects the overall viscosity of the emulsion, and its overall trend increases in proportion to the concentration. The dependence of apparent viscosity on the shear rate of emulsions prepared at different $\mathrm{pH}$ values is shown in Fig. 3h. The flow curves of emulsion samples under all pH value systems exhibited shear thinning, with the viscosity decreasing with the increasing of $\mathrm{pH}$ value. $\mathrm{A}$ lower $\mathrm{pH}$ can decrease the surface charge of CNC/LAE NRs. CNC/LAE NRs are more likely to be attracted and connected with each other to form a stronger network, either by surface-surface or edge-surface interactions. A higher $\mathrm{pH}$ can increase $\mathrm{OH}^{-}$and decrease $\mathrm{H}^{+}$, leading to a more negative surface charge of $\mathrm{CNC/LAE} \mathrm{NRs,} \mathrm{which} \mathrm{ends} \mathrm{with} \mathrm{a} \mathrm{larger} \mathrm{electrical} \mathrm{repulsive} \mathrm{force} \mathrm{among} \mathrm{particles.} \mathrm{In} \mathrm{this} \mathrm{case,} \mathrm{the}$ viscoelasticity of the interface layer is weak. Hence, the particles would connect with each other by dominated edge-edge particle interactions and form a weaker network. In addition, the highest viscosity among all the emulsions is exhibited by the as-prepared Pickering emulsions at $\mathrm{pH} 3$. These data indicate that interfacial packing behaviors and interactions of particles at the interface are affected by the $\mathrm{pH}$ conditions, which could result in a weaker network architecture and vitrified emulsions with poorer viscoelasticity at higher $\mathrm{pH}$ (i.e., $\mathrm{pH} \mathrm{7,9}$ and 12) (Zhou et al., 2018). Thus, ASA emulsions prepared at a $\mathrm{pH}$ range between 3 and 5 possessed a more favorable structure with gel-like elastic network. 


\subsection{Interfacial adsorption of LAE/CNC NRs}

Fig. 4a, b shows the CLSM of ASA emulsion, where red color refers to the dyed ASA oil with Nile red, and blue color refers to the emulsifier of LAE/CNC NRs. In addition, the blue color around the droplets refers to the flocculation consisted of LAE/CNC NRs, which is circled with deep orange and white color. Fig. 4c is the merged CLSM image. Interestingly, an incompletely covered ASA droplet with a black loophole (in Fig. 4b) and red oil phase inside (Fig. 4c) can be observed, suggesting that the LAE/CNC NRs jammed on the ASA-water interface instead of entering the oil phase. To observe the distribution of LAE/CNC NRs at the interface, the dosage of CNC NRs was reduced to $0.2 \%$, and the result is shown in Fig. $4 \mathrm{~d}$. It can be seen that the LAE/CNC NRs microgels with "fried-egg" structure tended to swell in the oil phase and partially collapse in the water phase with a contact angle $(\theta)$ favoring O/W emulsion-type stabilization (Rey, Fernandez-Rodriguez, Karg, Isa, \& Vogel, 2020). Under low concentrations of CNC NRs, such microgels would decrease the interfacial stability and lead to the aggregation of adjacent droplets, which resulted in large droplets and accelerated hydrolysis of ASA. The micro-flocculation formed by LAE/CNC NRs caused bridging flocculation between droplets, which could further prevent the coalescence and oil-phase transference between oil droplets (the elliptical area heighted by orange dot-line in Fig. 4b and 4e), facilitating the stability of emulsion obtained.

The total coverage rate $C(\%)$ of $\mathrm{LAE} / \mathrm{CNC}$ NRs on emulsion droplet was calculated by (Bai et al., 2018)

$$
C=\frac{m_{p} D}{6 h \rho V_{\text {oil }}}
$$

where $m_{p}$ is the mass of LAE/CNC NRs, $D$ is the average diameter of ASA droplets $\left(D_{43}\right), h$ is the thickness of LAE/CNC NRs (measured from AFM image), $\rho$ is the density of LAE/CNC NRs $\left(1.6 \mathrm{~g} / \mathrm{cm}^{3}\right)$, and $V_{\text {oil }}$ is the volume of ASA. It was calculated that $C$ was $64.75 \%$, with $0.02 \%$ of $L A E$ and $0.2 \% \mathrm{CNC}$. When the concentration of CNC increased by $2.0 \%, C$ was as high as $84.25 \%$, suggesting well coverage and stabilization of LAE/CNC on the ASA oil.

ASA is a specific oil phase with the ability of esterification reaction and is bound to react with polyhydric $\mathrm{CNC}$, thus modifying the morphology of particles and altering the performance of emulsion. The centrifugal stability of the ASA Pickering emulsion stabilized by LAE/CNC is shown in Fig. $5 \mathrm{a}$. The results show that the centrifugal stability of the emulsion increases firstly and then decreases with the increasing dosage of LAE with the maximum stability achieved at $0.02 \%$ LAE. This may be caused by the protective effect of LAE on hydroxyl groups of CNC, which provided LAE/CNC NRs with moderate wettability at the oil-water interface. However, excessive LAE may be obstacles for the reaction, and the high hydrophobicity of CNC is not conducive to the adsorption of LAE/CNC NRs, which ultimately reduces the stability of emulsion. AFM images of the emulsion stabilized by LAE/CNC NRs are shown in Fig. 5b, which shows that the LAE/CNC NRs form denser arrangement around the emulsion droplets with increasing dosage of LAE. The emulsion with $0.02 \%$ LAE has smaller droplet size and more uniform 
longitudinal distribution (-201.4 165.7 nm), which improve the adsorption stability of LAE/CNC NRs at the water-oil interface.

Fig. 5c displays the SEM image of ASA emulsion stabilized by CNC solely. The granular film structure with honeycomb-like shape is revealed, which may be caused by the aligned arrangement of CNC. It is considered that this arrangement mode may be related to the decreased repulsion between ASA-CNC and CNC caused by reduced amount of hydroxyl groups. Otherwise, such phenomenon of alignment is more obvious without LAE in TEM image (Fig. 5d), where CNC sufficiently reacts with ASA and forms NRs matrix with oriented arrangement (white dotted box). Whereas, the ASA emulsion stabilized by LAE/CNC NRs presents a circular arc granular film in Fig. $5 e$, due to the effective stabilizing and protecting function of LAE/CNC on ASA droplets by valid coverage. Moreover, the uniformly distributed nanorods on the emulsion's surface can be clearly observed in Fig. $5 f$. We assume that LAE protects the hydroxyl groups of CNC from being completely esterified, thereby presenting NRs film with anisotropic arrangement, allowing the occurrence of chemical crosslinking between parts of hydroxyl groups on LAE/CNC and carbonyl lactone on ASA at the o/w interface. The LAE/CNC NRs with moderate wettability induced by the esterification could form microgels at the o/w interface, which is consistent with the results observed in Fig. 3d.

The schematic diagram of chemical cross-linking process and the stabilizing effect of LAE/CNC NRs at the ASA-water interface is drawn and displayed in Fig. $5 \mathrm{~g}$, h. CNC can sufficiently react with ASA and generate hydrophobic ASA-CNC (blue) at the ASA-water interface with little LAE. Then the ASA is emulsified as oil in water emulsion by interfacial nanoparticles along with CNC NRs in continuous phase. However, the ASA emulsion shows poor stability and easily forms honeycomb-like walls and oriented CNC because of the coalescence and compression of droplets. To some extent, the introduction of LAE restricts the reaction between ASA and CNC, and more LAE/CNC NRs are cross-linked with LAE and form stable interfacial granular film, which leads to the formation of gel state with moderate wettability of LAE/CNC NRs. The chemical crosslinking between LAE/CNC and ASA can efficiently prevent droplet coalescence induced by phase migration, hinder hydrolyzation of ASA and improve the stability of emulsion.

In view of the characteristic functional group, the ASA emulsion shows a high intensity at $1863 \mathrm{~cm}^{-1}$, $1778 \mathrm{~cm}^{-1}$, and essentially unchanged intensity at $1663 \mathrm{~cm}^{-1}$ after $6 \mathrm{~h}$ in Fig. $6 \mathrm{a}$, which are assigned to the carbonyl lactone ring of ASA and carboxyl group of ASA-acid, respectively. These peaks show significant changes until $12 \mathrm{~h}$ later. After about $144 \mathrm{~h}$, ASA started to lose most of the activity (Fig. S6). It is concluded that LAE/CNC NRs had a lasting protective ability against ASA and significantly inhibited the hydrolysis reaction of ASA within $12 \mathrm{~h}$, which will promote the sizing application of cellulose-fiber paper.

Furthermore, the sizing performance of ASA sizing Pickering emulsion is evaluated with sizing degree and the contact angle of the sized paper. The dynamic water contact angle of the sized paper is shown in Fig. $6 \mathrm{~b}$, and the contact angle gradually increases with the increasing amount of CNC added in ASA 
emulsion, which indicates the improved hydrolysis resistance. The ASA sized paper has a contact angle of $115^{\circ}$ after retaining water for $1 \mathrm{~s}$ with $2 \%$ CNC NRs, while the contact angle is $103^{\circ}$ for the sized paper with $0.5 \%$ CNC NRs. Even after $900 \mathrm{~s}$, the contact angle of the paper sized by ASA emulsion containing $0.5 \%$ and $2.0 \% \mathrm{LAE} / \mathrm{CNC}$ NRs decreased slowly to $72^{\circ}$ and $93^{\circ}$ respectively, which further confirms the reinforcement of LAE/CNC NRs. Fig. $6 \mathrm{c}$ shows that the sizing degree improves with increasing dosage of ASA emulsion, and the sizing degree increases by $152 \mathrm{~s}$ with $0.5 \%$ ASA (relative to oven-dried stock), and then increases by about $300 \mathrm{~s}$ with $1.0 \%$ ASA. However, the static contact angle of sized paper increases firstly and then decreases with the increasing dosage of ASA, which reaches a maximum value of $113^{\circ}$ with the ASA emulsion containing 1.0\% CNC NRs. Overall, the ASA emulsion has good internal sizing performance. The micro-CT of ASA emulsion sized paper shows great differences with different emulsifiers in Fig. $6 \mathrm{~d}$. The blue and green colors refer to a lot of hydrophilic hydroxyl groups and hydrolyzed ASA acid are remained with ASA emulsion stabilized by CNC NRs solely. Whereas, the golden color refers to a lot of ASA-celluloses, which further explains the enhancement of LAE on the sizing performance of ASA emulsion.

Fig. 7. illustrates the adsorption of LAE/CNC NRs, the chemical cross-linking between LAE/CNC NRs and ASA at the ASA-water interface, and the stabilization mechanism of Pickering emulsion. Otherwise, the interfacial granular film composed of amphiphilic LAE/CNC NRs prevents the migration of oil phase and the coalescence of droplets, reducing the contact and hydrolysis reaction between water and ASA. Moreover, LAE/CNC NRs could be crosslinked with ASA and ASA-acid, generating a more compact interface granular film and improving the stability of emulsion. At present, CNC has been applied to many areas, where cationic surfactants are commonly involved, so it is very important to understand this binding interaction. The binding interactions between $\mathrm{CNC}$ and these oppositely charged surfactants could have a profound influence on the colloidal stability and functionality of CNC in these systems, as well as the bulk properties of the systems. We provide a sustainable and ecologically compatible surface modification strategy for $\mathrm{CNC}$ by the combination of a novel biologically-derived surfactant and a simple environmental benign mixing approach. The successful formulation of LAE/CNC-stabilized ASA Pickering emulsion provides a basis for the application of ASA paper sizing system with tunable and functional properties based on Pickering systems.

\section{Conclusion}

This study investigated the dual-enhancement effect of interfacial adsorption and chemical crosslinking on the emulsification properties of the LAE/CNC NRs in ASA Pickering emulsions. Moderate wettability of $\mathrm{CNC}$ with a contact angle of $87.6^{\circ}$ can be achieved by the introduction of $L A E$, which facilitates the attachment on the surface of ASA droplets, and generates stable emulsions with uniform, small and spherical droplets. The LAE/CNC-ASA droplets exhibit a small droplet size, long-term storge stability and strong resistance to flocculation when $\mathrm{pH}$ is below 5. CLSM images showed that the LAE/CNC are adsorbed at the oil-water interface. SEM and TEM images revealed that the ASA Pickering emulsion droplets were encircled and stabilized by matrix of LAE/CNC NRs, which were densely immobilized at the oil-water interface. The chemical crosslinking between LAE/CNC and ASA can efficiently prevent droplet 
coalescence induced by phase migration, hinder hydrolyzation of ASA and improve the stability of emulsion. LAE/CNC NRs had a lasting protective ability against ASA and significantly inhibited the hydrolysis reaction of ASA within $12 \mathrm{~h}$. Sized cellulose paper with LAE/CNC-ASA emulsion conducted 300 $s$ of sizing-degree with $113^{\circ}$ of water contact angle. These findings offer significant insights into the fabrication of the surfactant-modified CNC and the commercial application of the LAE/CNC NRsstabilized ASA Pickering emulsions in the papermaking industry.

\section{Declarations}

competing interest

There are no conflicts of interest to declare.

\section{CRediT authorship contribution statement}

Yucheng Li: Methodology, Investigation, Software, Methodology, Data Curation, Formal Analysis, Writing Original Draft. Dehai Yu: Conceptualization, Supervision, Investigation, Writing - Review \& Editing. Xiangyu Wang: Investigation. Qiang Wang: Methodology. Zhen Zhang: Resources. Wenxia Liu: Supervision, Writing - Review \& Editing.

\section{Acknowledgements}

This work was supported by the project ZR2020MB128 supported by Shandong Provincial Natural Science Foundation Natural Science Foundation; the Foundation of Guangxi Key Laboratory of Clean Pulp \& Papermaking and Pollution Control (Grant No. 2019KF08), College of Light Industry and Food Engineering, Guangxi University; the Foundation (Grant No. 2020KFJJ11) of National Forestry and Grassland Administration Key Laboratory of Plant Fiber Functional Materials; the Outstanding Youth Innovation Team Project of Shandong Provincial University; the Special Project for Integration of Science, Education, and Industry (Grant No. 2020KJC-ZD06).

\section{Appendix A. Supplementary data}

Supplementary material related to this article can be found, in the online version, at doi:

\section{References}

Bai, L., Huan, S., Zhu, Y., Chu, G., McClements, D. J., \& Rojas, O. J. (2021). Recent advances in food emulsions and engineering foodstuffs using plant-based nanocelluloses. Annual Review of Food Science and Technology, 12, 383-406. https://doi.org/10.1146/annurev-food-061920-123242

Bai, L., Xiang, W., Huan, S., \& Rojas, O. J. (2018). Formulation and stabilization of concentrated edible oilin-water emulsions based on electrostatic complexes of a food-grade cationic surfactant (ethyl lauroyl 
arginate) and cellulose nanocrystals. Biomacromolecules, 19(5), 1674-1685.

https://doi.org/10.1021/acs.biomac.8b00233

Ben Azouz, K., Ramires, E. C., Van den Fonteyne, W., El Kissi, N., \& Dufresne, A. (2012). Simple method for the melt extrusion of a cellulose nanocrystal reinforced hydrophobic polymer. ACS Macro Letters, 1(1), 236-240. https://doi.org/10.1021/mz2001737

Binks, B. P., Muijlwijk, K., Koman, H., \& Poortinga, A. T. (2017). Food-grade Pickering stabilisation of foams by in situ hydrophobisation of calcium carbonate particles. Food Hydrocolloids, 63, 585-592. https://doi.org/10.1016/j.foodhyd.2016.10.002

Bollhorst, T., Rezwan, K., \& Maas, M. (2017). Colloidal capsules: nano-and microcapsules with colloidal particle shells. Chemical Society Reviews, 46(8), 2091-2126. https://doi.org/10.1039/C6CS00632A

Capron, I., Rojas, O. J., \& Bordes, R. (2017). Behavior of nanocelluloses at interfaces. Current Opinion in Colloid \& Interface Science, 29, 83-95. https://doi.org/10.1016/j.cocis.2017.04.001

Chen, Q.-H., Liu, T.-X., \& Tang, C.-H. (2019). Tuning the stability and microstructure of fine Pickering emulsions stabilized by cellulose nanocrystals. Industrial Crops and Products, 141, 111733.

https://doi.org/10.1016/j.indcrop.2019.111733

Chi, K., \& Catchmark, J. M. (2017). Crystalline nanocellulose/lauric arginate complexes. Carbohydrate Polymers, 175, 320-329. https://doi.org/10.1016/j.carbpol.2017.08.005

Chu, Y., Sun, Y., Wu, W., \& Xiao, H. (2020). Dispersion properties of nanocellulose: a review. Carbohydrate Polymers, 116892. https://doi.org/10.1016/j.carbpol.2020.116892

Cunha, A. G., Mougel, J.-B., Cathala, B., Berglund, L. A., \& Capron, I. (2014). Preparation of double Pickering emulsions stabilized by chemically tailored nanocelluloses. Langmuir, 30(31), 9327-9335. https://doi.org/10.1021/la5017577

Dickinson, E. (2017). Biopolymer-based particles as stabilizing agents for emulsions and foams. Food Hydrocolloids, 68, 219-231. https://doi.org/10.1016/j.foodhyd.2016.06.024

Du, W., Guo, J., Li, H., \& Gao, Y. (2017). Heterogeneously modified cellulose nanocrystals-stabilized pickering emulsion: preparation and their template application for the creation of PS microspheres with amino-rich surfaces. ACS Sustainable Chemistry \& Engineering, 5(9), 7514-7523.

https://doi.org/10.1021/acssuschemeng.7b00375

Gong, X., Wang, Y., \& Chen, L. (2017). Enhanced emulsifying properties of wood-based cellulose nanocrystals as Pickering emulsion stabilizer. Carbohydrate Polymers, 169, 295-303. https://doi.org/10.1016/j.carbpol.2017.04.024 
Habibi, Y., Lucia, L. A., \& Rojas, O. J. (2010). Cellulose nanocrystals: chemistry, self-assembly, and applications. Chemical reviews, 110(6), 3479-3500. https://doi.org/10.1021/cr900339w

Hong, C.-S., Park, J. H., Lee, S., Rhoo, K. Y., Lee, J. T., \& Paik, S. R. (2018). Fabrication of protease-sensitive and light-responsive microcapsules encompassed with single layer of gold nanoparticles by using selfassembly protein of a-synuclein. ACS applied materials \& interfaces, 10(31), 26628-26640.

https://doi.org/10.1021/acsami.8b07661

Huang, L., Ye, Z., \& Berry, R. (2016). Modification of cellulose nanocrystals with quaternary ammoniumcontaining hyperbranched polyethylene ionomers by ionic assembly. ACS Sustainable Chemistry \& Engineering, 4(9), 4937-4950. https://doi.org/10.1021/acssuschemeng.6b01253

Hubbe, M. A. (2007). Paper's resistance to wetting-A review of internal sizing chemicals and their effects. BioResources, 2(1), 106-145.

Johnson, R. K., Zink-Sharp, A., \& Glasser, W. G. (2011). Preparation and characterization of hydrophobic derivatives of TEMPO-oxidized nanocelluloses. Cellulose, 18(6), 1599-1609.

https://doi.org/10.1007/s10570-011-9579-y

Kedzior, S. A., Gabriel, V. A., Dubé, M. A., \& Cranston, E. D. (2020). Nanocellulose in emulsions and heterogeneous water-based polymer systems: A review. Advanced Materials, 2002404. https://doi.org/10.1002/adma.202002404

Li, G., Song, Z., Liu, W., Yu, D., \& Wang, H. (2017). Alkyl ketene dimer emulsions stabilized by layered double hydroxide particles modified with glutamic acid. Industrial \& Engineering Chemistry Research, 56(40), 11435-11442. https://doi.org/10.1021/acs.iecr.7b02532

Li, Y., Liu, X., Zhang, Z., Zhao, S., Tian, G., Zheng, J., . . Russell, T. P. (2018). Adaptive structured pickering emulsions and porous materials based on cellulose nanocrystal surfactants. Angewandte Chemie International Edition, 57(41), 13560-13564. https://doi.org/10.1002/anie.201808888

Li, Y., Zhao, R., Hu, F., Lu, P., Ji, D., Luo, Q., . . Song, Z. (2021). Laponite/lauric arginate stabilized AKD Pickering emulsions with shell-tunable hydrolytic resistance for use in sizing paper. Applied Clay Science, 206, 106085. https://doi.org/10.1016/j.clay.2021.106085

Lu, Z., Huang, J., Songfeng, E., Li, J., Si, L., Yao, C., . . Zhang, M. (2020). All cellulose composites prepared by hydroxyethyl cellulose and cellulose nanocrystals through the crosslink of polyisocyanate.

Carbohydrate Polymers, 250, 116919. https://doi.org/10.1016/j.carbpol.2020.116919

McClements, D. J., \& Gumus, C. E. (2016). Natural emulsifiers-Biosurfactants, phospholipids, biopolymers, and colloidal particles: Molecular and physicochemical basis of functional performance. Advances in Colloid and interface Science, 234, 3-26. https://doi.org/10.1016/j.cis.2016.03.002 
Mohit, T., Bhadra, K., Goswami, S., \& Agarwal, N. (2007). Successful trials \& optimization of ASA sizing. IPPTA, 19(4), 143.

Niu, F., Han, B., Fan, J., Kou, M., Zhang, B., Feng, Z.-J., . . Zhou, W. (2018). Characterization of structure and stability of emulsions stabilized with cellulose macro/nano particles. Carbohydrate Polymers, 199, 314-319. https://doi.org/10.1016/j.carbpol.2018.07.025

Patel, A. S., Lakshmibalasubramaniam, S., Nayak, B., \& Camire, M. E. (2021). Lauric acid adsorbed cellulose nanocrystals retained the physical stability of oil-in-water Pickering emulsion during different dilutions, pH, and storage periods. Food Hydrocolloids, 107139.

https://doi.org/10.1016/j.foodhyd.2021.107139

Pickering, S. (1907). Cxcvi. J. Chem. Soc., Trans, 91(0), 2001-2021.

Qiao, X., Wang, L., Shao, Z., Sun, K., \& Miller, R. (2015). Stability and rheological behaviors of different oil/water emulsions stabilized by natural silk fibroin. Colloids and Surfaces A: Physicochemical and Engineering Aspects, 475, 84-93. https://doi.org/10.1016/j.colsurfa.2015.01.094

Rey, M., Fernandez-Rodriguez, M. A., Karg, M., Isa, L., \& Vogel, N. (2020). Poly-N-isopropylacrylamide nanogels and microgels at fluid interfaces. Accounts of chemical research, 53(2), 414-424.

https://doi.org/10.1021/acs.accounts.9b00528

Robert, J. (1990). Overview and theories of neutral and alkaline sizing. In: Neutral/Alkaline Papermaking: TAPPI Short Course Notes.

Saidane, D., Perrin, E., Cherhal, F., Guellec, F., \& Capron, I. (2016). Some modification of cellulose nanocrystals for functional Pickering emulsions. Philosophical Transactions of the Royal Society A: Mathematical, Physical and Engineering Sciences, 374(2072), 20150139.

https://doi.org/10.1098/rsta.2015.0139

Scatena, L., Brown, M., \& Richmond, G. (2001). Water at hydrophobic surfaces: weak hydrogen bonding and strong orientation effects. Science, 292(5518), 908-912. https://doi.org/10.1126/science.1059514

Sun, G., Liu, X., McClements, D. J., Liu, S., Li, B., \& Li, Y. (2021). Chitin nanofibers improve the stability and functional performance of Pickering emulsions formed from colloidal zein. Journal of Colloid and Interface Science, 589, 388-400. https://doi.org/10.1016/j.jcis.2021.01.017

Tan, H., Liu, W., Yu, D., Li, H., Hubbe, M. A., Gong, B., . . Li, G. (2014). ASA-in-water emulsions stabilized by laponite nanoparticles modified with tetramethylammonium chloride. Chemical Engineering Science, 116, 682-693. https://doi.org/10.1016/j.ces.2014.06.005

Tang, J., Lee, M. F. X., Zhang, W., Zhao, B., Berry, R. M., \& Tam, K. C. (2014). Dual responsive pickering emulsion stabilized by poly [2-(dimethylamino) ethyl methacrylate] grafted cellulose nanocrystals. Biomacromolecules, 15(8), 3052-3060. https://doi.org/10.1021/bm500663w 
Tardy, B. L., Yokota, S., Ago, M., Xiang, W., Kondo, T., Bordes, R., \& Rojas, O. J. (2017). Nanocellulosesurfactant interactions. Current Opinion in Colloid \& Interface Science, 29, 57-67.

https://doi.org/10.1016/j.cocis.2017.02.004

Thaiphanit, S., Schleining, G., \& Anprung, P. (2016). Effects of coconut (Cocos nucifera L.) protein hydrolysates obtained from enzymatic hydrolysis on the stability and rheological properties of oil-in-water emulsions. Food Hydrocolloids, 60, 252-264. https://doi.org/10.1016/j.foodhyd.2016.03.035

Tiong, A. C. Y., Tan, I. S., Foo, H. C. Y., Lam, M. K., Mahmud, H. B., \& Lee, K. T. (2020). Macroalgae-derived regenerated cellulose in the stabilization of oil-in-water Pickering emulsions. Carbohydrate Polymers, 249, 116875. https://doi.org/10.1016/j.carbpol.2020.116875

Wang, J., Deng, H., Sun, Y., \& Yang, C. (2020). Montmorillonite and alginate co-stabilized biocompatible Pickering emulsions with multiple-stimulus tunable rheology. Journal of Colloid and Interface Science, 562, 529-539. https://doi.org/10.1016/j.jcis.2019.11.081

Xia, Y., Wu, J., Wei, W., Du, Y., Wan, T., Ma, X., . . Yue, H. (2018). Exploiting the pliability and lateral mobility of Pickering emulsion for enhanced vaccination. Nature materials, 17(2), 187-194. https://doi.org/10.1038/nmat5057

Yao, M., Liu, Y., Qin, C., Meng, X., Cheng, B., Zhao, H., . . Huang, Z. (2021). Facile fabrication of hydrophobic cellulose-based organic/inorganic nanomaterial modified with POSS by plasma treatment. Carbohydrate Polymers, 253, 117193. https://doi.org/10.1016/j.carbpol.2020.117193

Yu, D., Li, G., Kong, F., Wang, H., Liu, W., Song, Z., .. . Zhao, J. R. (2020). Encapsulation of alkenylsuccinic anhydride oil droplets in Laponite nanoparticles modified by carbon nitride quantum dots: Enhancement of emulsion stability and paper sizing performance. Applied Clay Science, 191, 105608. https://doi.org/10.1016/j.clay.2020.105608

Yu, D., Lin, Z., \& Li, Y. (2013). Octadecenylsuccinic anhydride Pickering emulsion stabilized by $\mathrm{Y}^{-}$ methacryloxy propyl trimethoxysilane grafted montmorillonite. Colloids and Surfaces A: Physicochemical and Engineering Aspects, 422, 100-109. https://doi.org/10.1016/j.clay.2020.105608

Zhang, W., Gu, X., Liu, X., \& Wang, Z. (2021). Fabrication of Pickering emulsion based on particles combining pectin and zein: Effects of pectin methylation. Carbohydrate Polymers, 256, 117515. https://doi.org/10.1016/j.carbpol.2020.117515

Zhang, Y., Zhu, G., Dong, B., Wang, F., Tang, J., Stadler, F. J., . . Xing, F. (2021). Interfacial jamming reinforced Pickering emulgel for arbitrary architected nanocomposite with connected nanomaterial matrix. Nature Communications, 12(1), 1-9. https://doi.org/10.1038/s41467-020-20299-6

Zhou, F., Zeng, T., Yin, S., Tang, C., Yuan, D., \& Yang, X. (2018). Development of antioxidant gliadin particle stabilized Pickering high internal phase emulsions (HIPEs) as oral delivery systems and the in vitro 
Zoppe, J. O., Venditti, R. A., \& Rojas, O. J. (2012). Pickering emulsions stabilized by cellulose nanocrystals grafted with thermo-responsive polymer brushes. Journal of Colloid and Interface Science, 369(1), 202209. https://doi.org/10.1016/j.jcis.2011.12.011

\section{Scheme}

Please see the Supplementary Files for the Scheme 1.

\section{Figures}

(a)

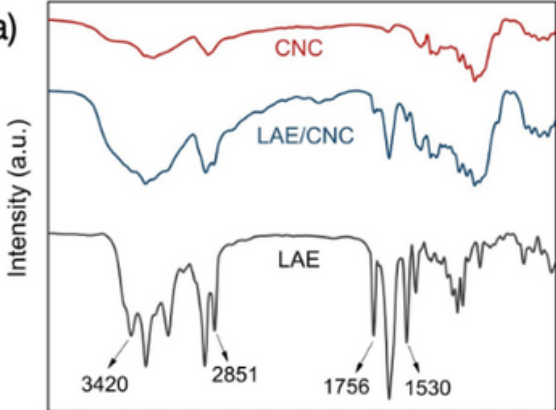

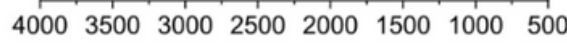

Wavenumber $\left(\mathrm{cm}^{-1}\right)$
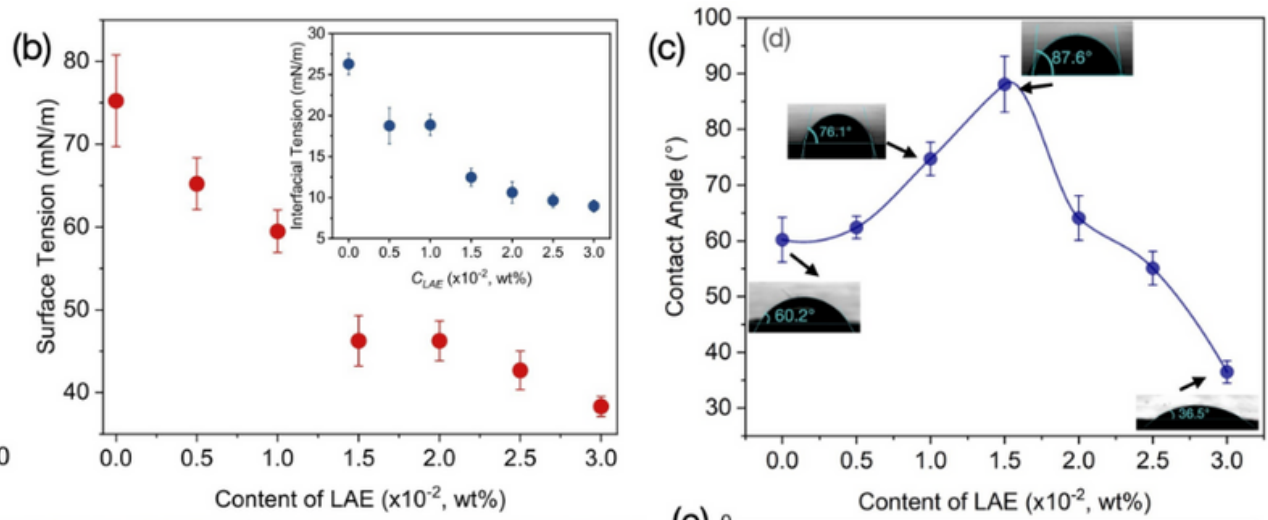

(d)
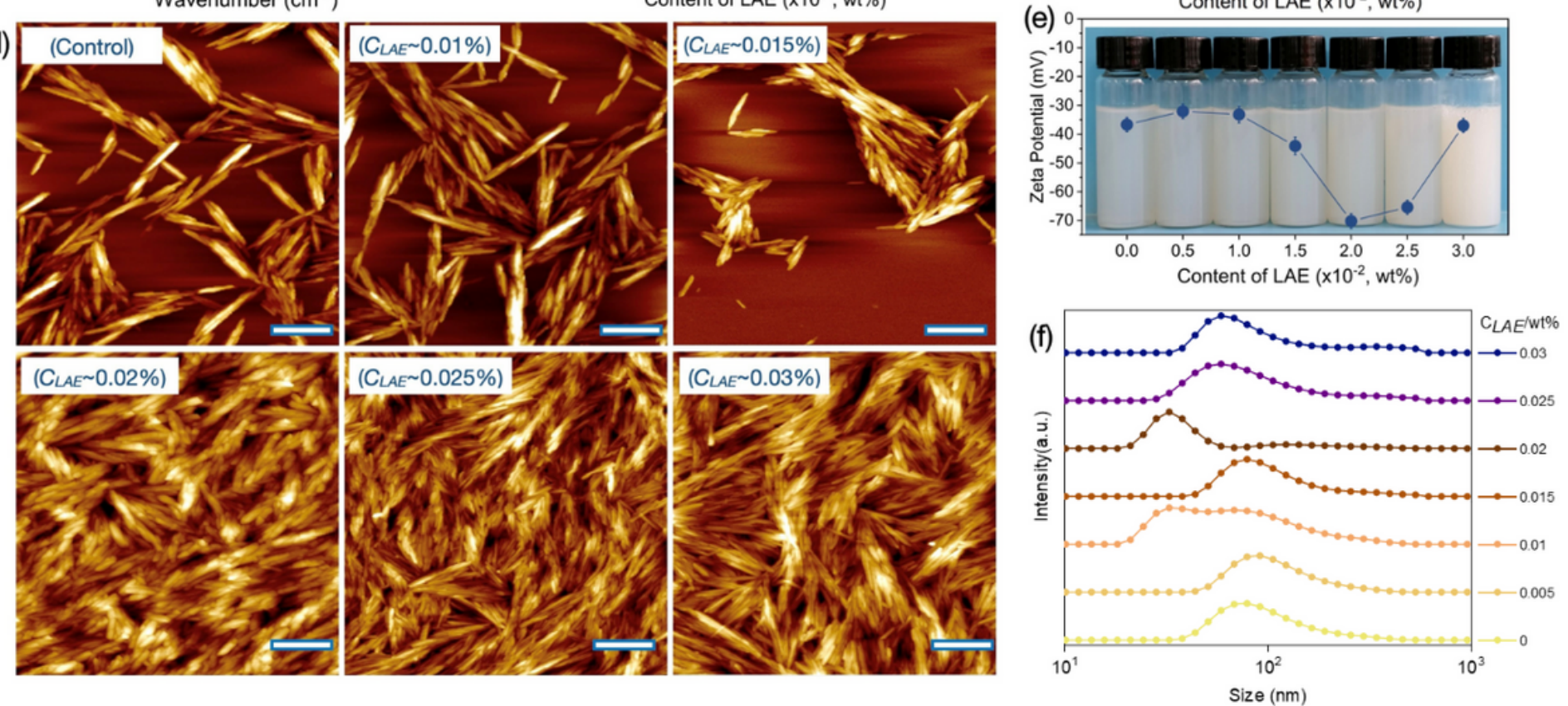

Figure 1

(a) FT-IR spectra of LAE, CNC, and LAE/CNC nanorods. (b) Surface tension and interface tension of $2.0 \%$ $\mathrm{CNC}$ aqueous dispersions containing different amounts of $\mathrm{LAE}$ at $25{ }^{\circ} \mathrm{C}$. (c) The three-phase contact angle of LAE/CNC as a function of initial surfactant content. (d) AFM height images of pristine CNC and $\mathrm{CNC} / \mathrm{LAE}$ nanorods with different content of $\mathrm{LAE}\left(C_{\mathrm{LAE}}\right)$. The CNC initial concentration was $2.0 \%$. All the 
scale bars are $200 \mathrm{~nm}$. (e) Zeta potential of LAE/CNC nanorod dispersions with different $C_{\text {LAE. }}$ (f) Particle distribution of $\mathrm{LAE} / \mathrm{CNC}$ with different $C_{\text {LAE. }}$
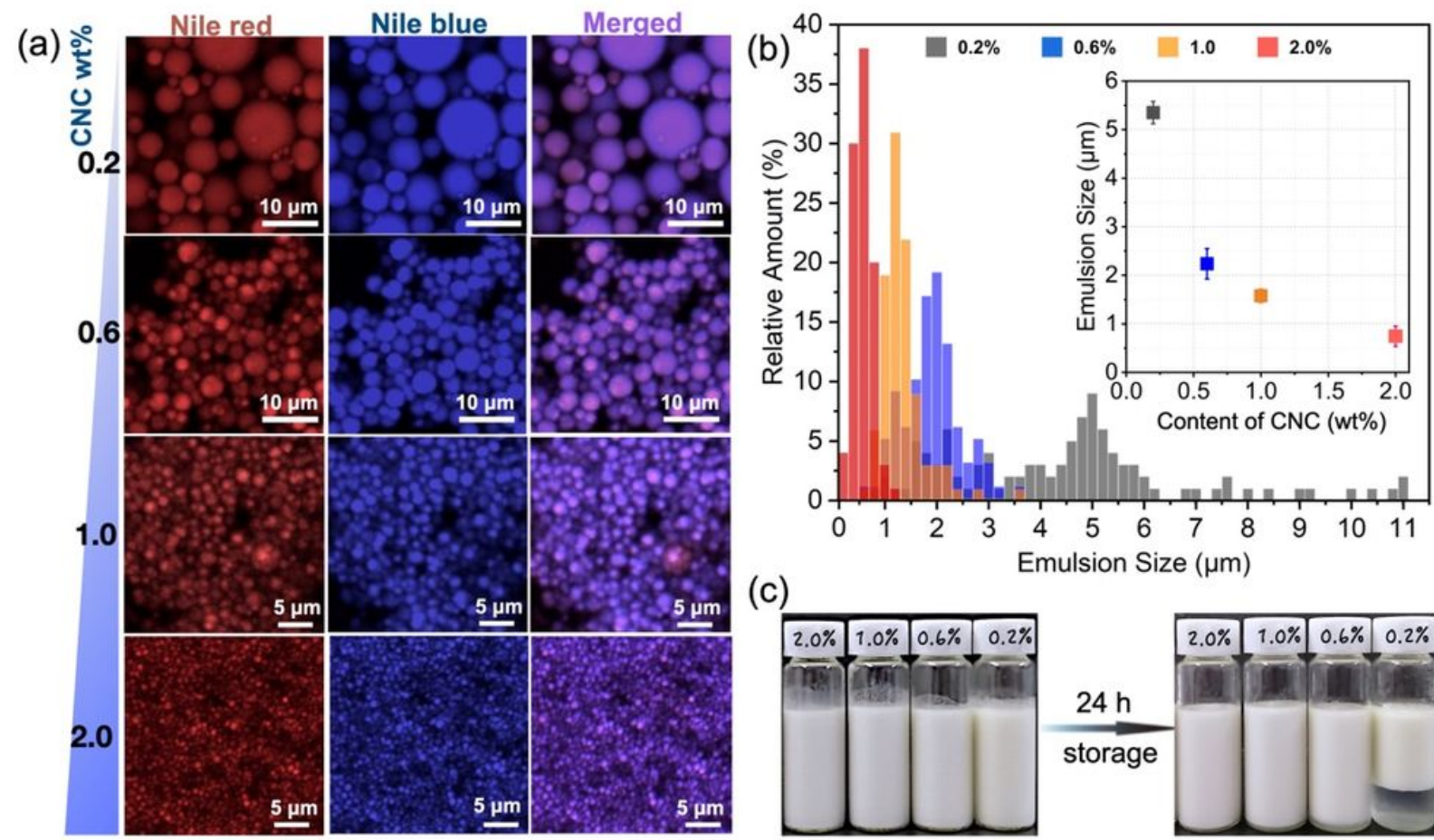

(c)
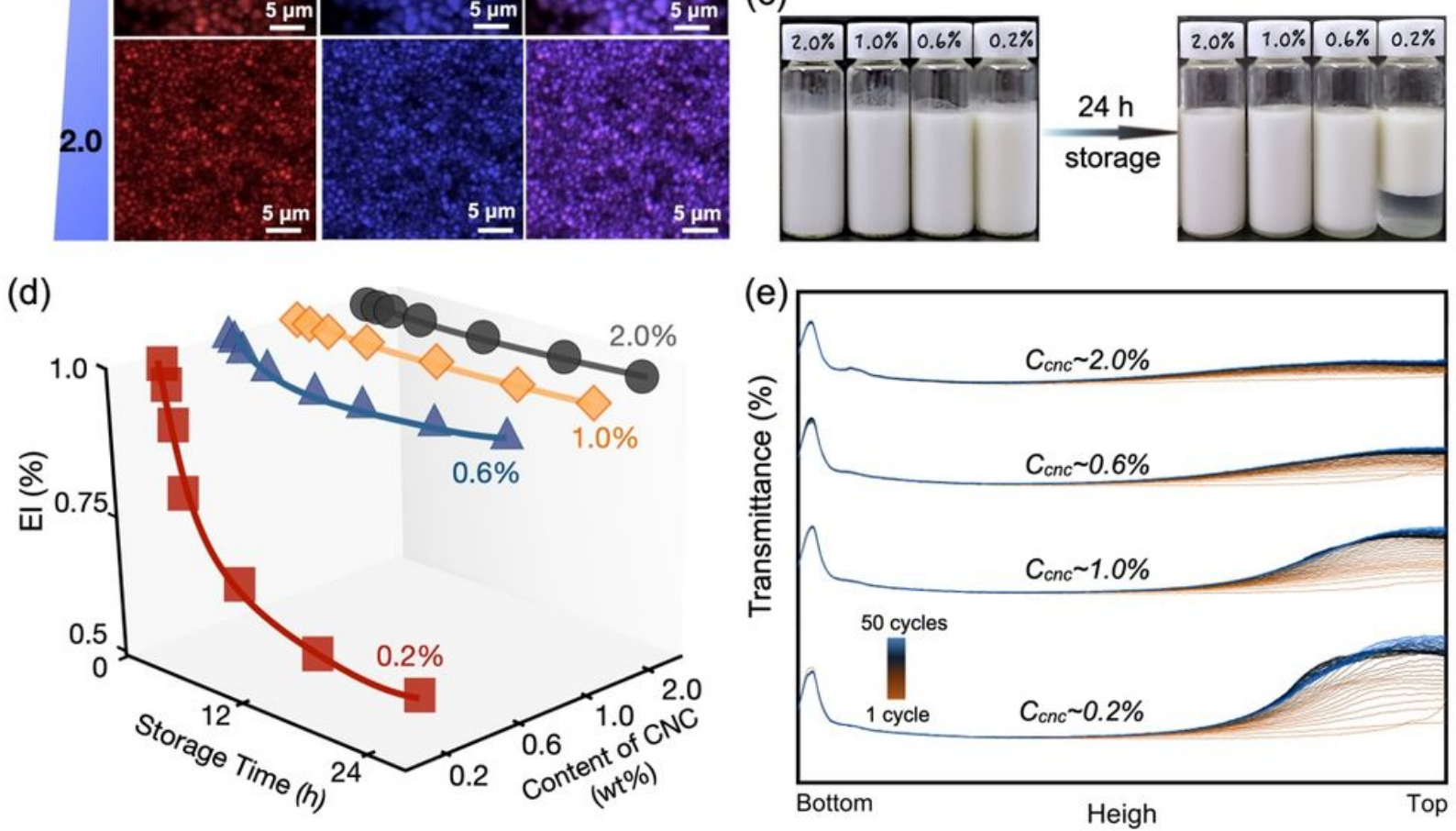

\section{Figure 2}

(a) CLSM images of LAE/CNC NRs-stabilized ASA Pickering emulsions with different CNC content $\left(C_{C N C}\right)$. All scale bars are $10 \mu \mathrm{m}$. (b) Particle size distribution of ASA emulsion droplets prepared by LAE/CNC NRs. Insert is the average droplet size spectra of the most probable sizes as a function of the $C_{C N C}$. (c) 
Appearance pictures of the ASA emulsions prepared after $24 \mathrm{~h}$ storage time. (d) Emulsion index of ASA emulsion at different storage times in panel c. (e) Transmittance profiles of as-prepared ASA emulsions containing $0.02 \%$ LAE and $0.2 \%$ 2.0\% CNC (water phase based) after 50 cycles of centrifugation at 2000 rpm. The horizontal axis indicates the height position along the test tube. All the samples were kept undisturbed at ambient temperature.

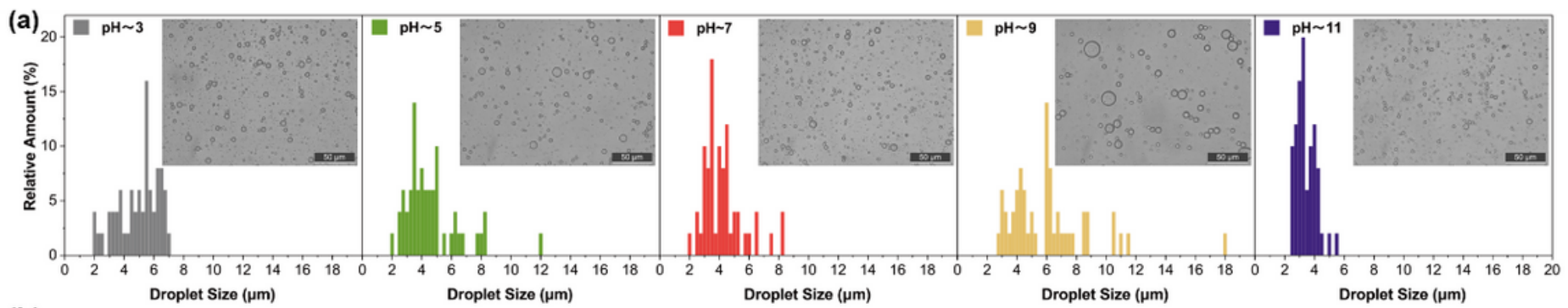

(b)

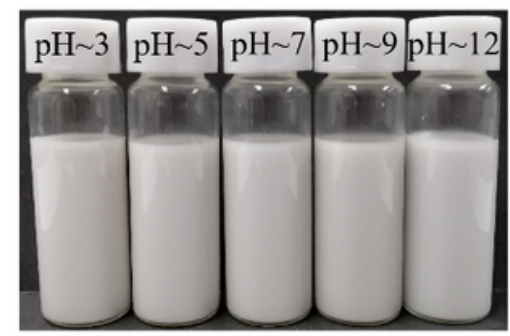

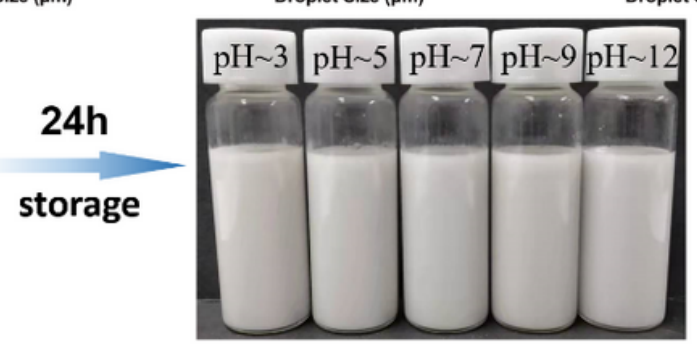

(c)
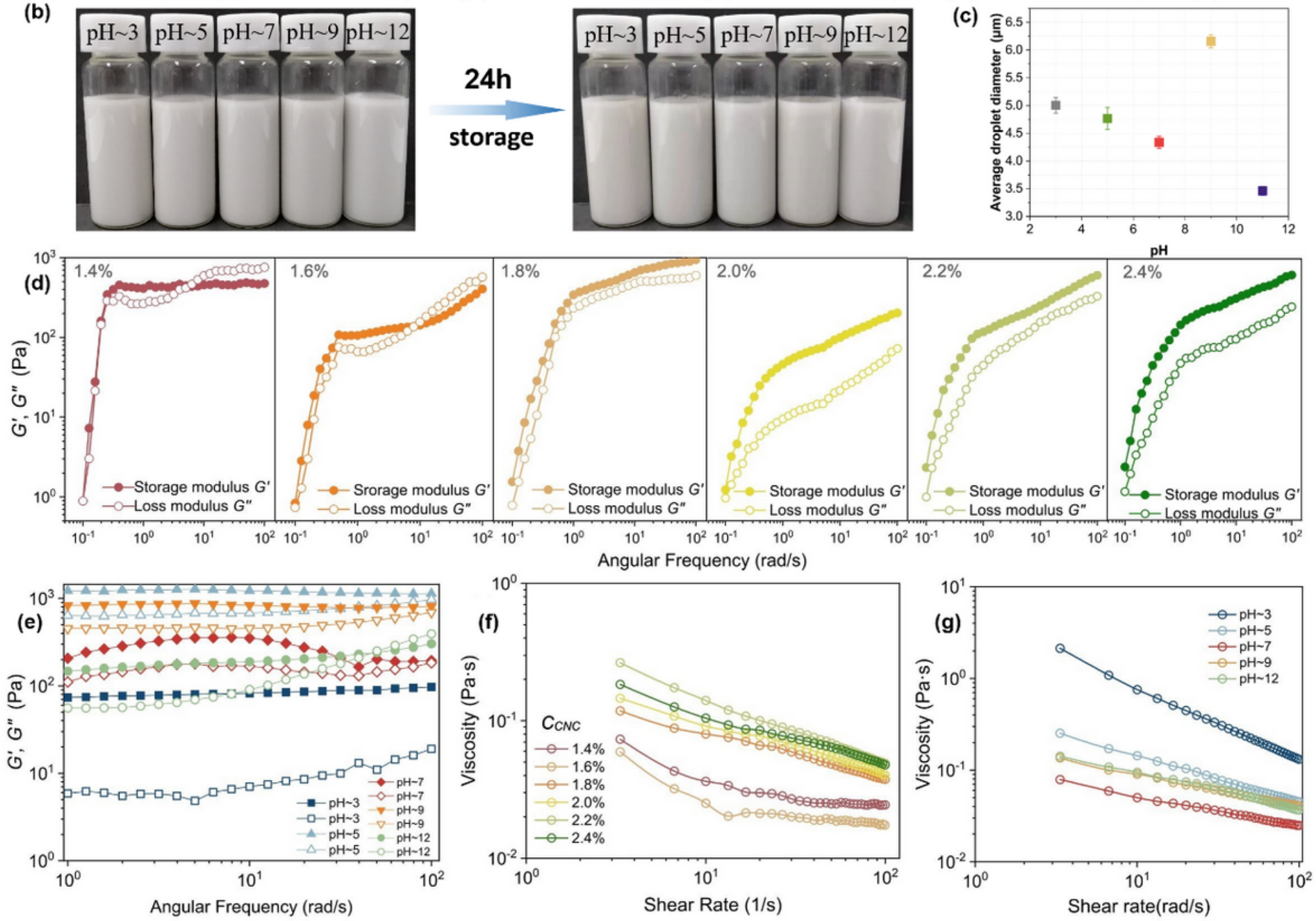

Figure 3

(a) Droplet size distribution of the ASA emulsion stabilized by LAE/CNC under different $\mathrm{pH}$ values. The insert is the relevant optical micrograph. All the scale bases are $50 \mu \mathrm{m}$. (b) Digital picture of the emulsion freshly prepared and stored for $24 \mathrm{~h}$. (c) The average droplet size versus $\mathrm{pH}$ value. Storage modulus ( $G^{\prime}$, filled symbols), and loss modulus ( $G$ ", open symbols), as a function of angular frequency for ASA 
Pickering emulsions (oil/water $=1: 2, \mathrm{v} / \mathrm{v}$ ) stabilized by LAE/CNC with various CNC concentrations $(\mathrm{d})$ and $\mathrm{pH}$ value (e). Flow profiles for ASA Pickering emulsions containing CNC at different concentrations ( $f$ ) and $\mathrm{pH}$ value $(\mathrm{g})$. LAE concentration fixed on $0.02 \%$ (based on aqueous phase).

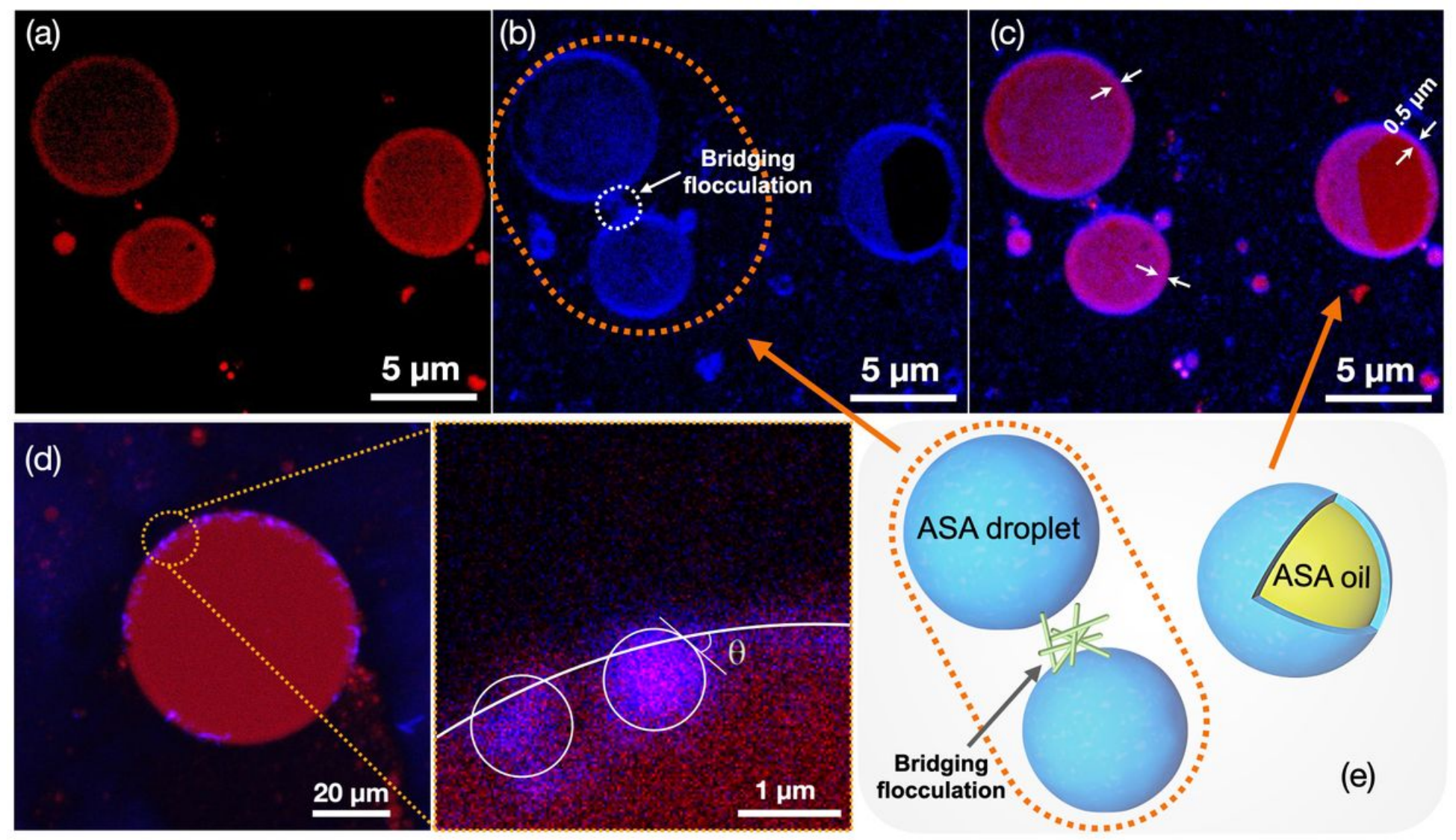

\section{Figure 4}

CLSM images of ASA Pickering emulsion stabilized by LAE/CNC nanorods with $0.5 \%$ CNC and $0.02 \%$ LAE. (a) Nile Red, (b) Nile Blue and (c) Merged. The orange and white dotted circles in panel b are used to highlight the oil droplets and particles, respectively. (d) Confocal microscopy image showing a close-up of a latex particle (blue) with a radius of 0.5 um at the surface of a large droplet of the ASA phase (red) in a matrix of the LAE/CNC water phase. The surface of the particle and the ASA/water interface are indicated by white lines and the contact angle is shown. (e) Schematic illustration of ASA droplets coated by LAE/CNC nanorods in panel $b$ and $c$. 

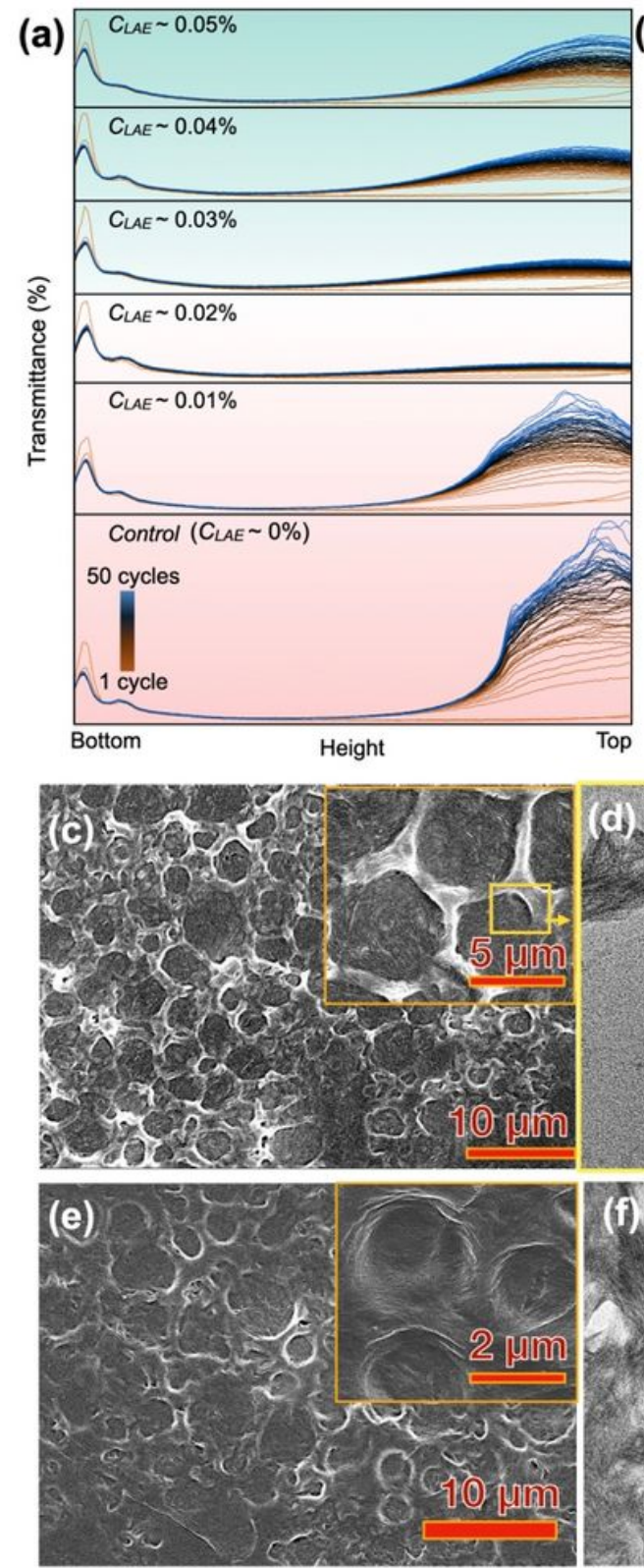

(b)
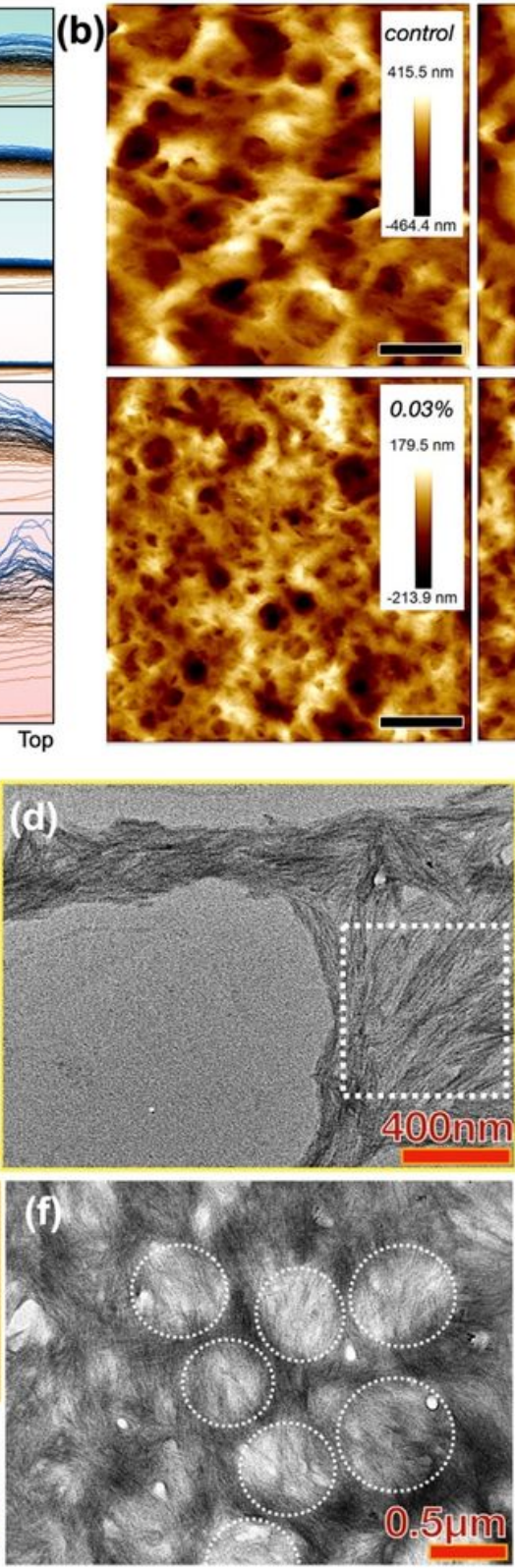

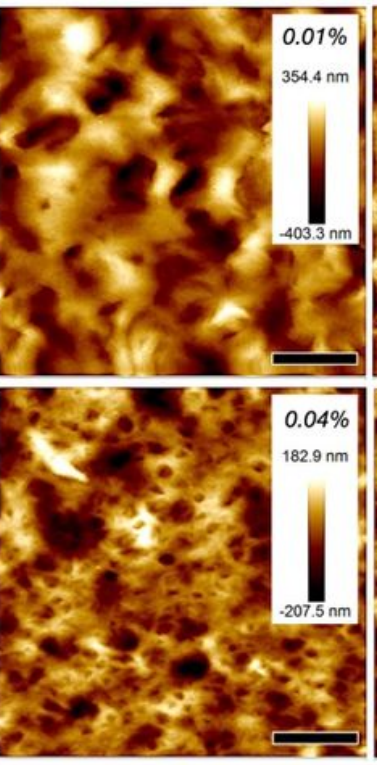

(g)

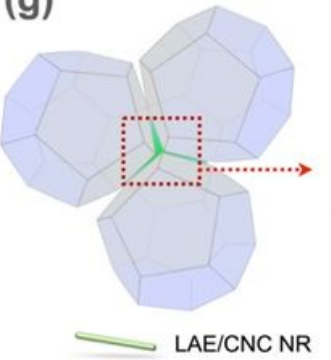

(h)

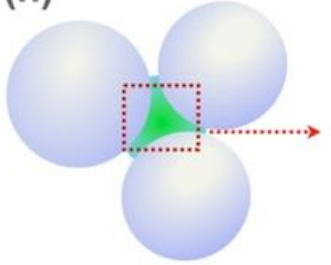

LAE/CNC NR
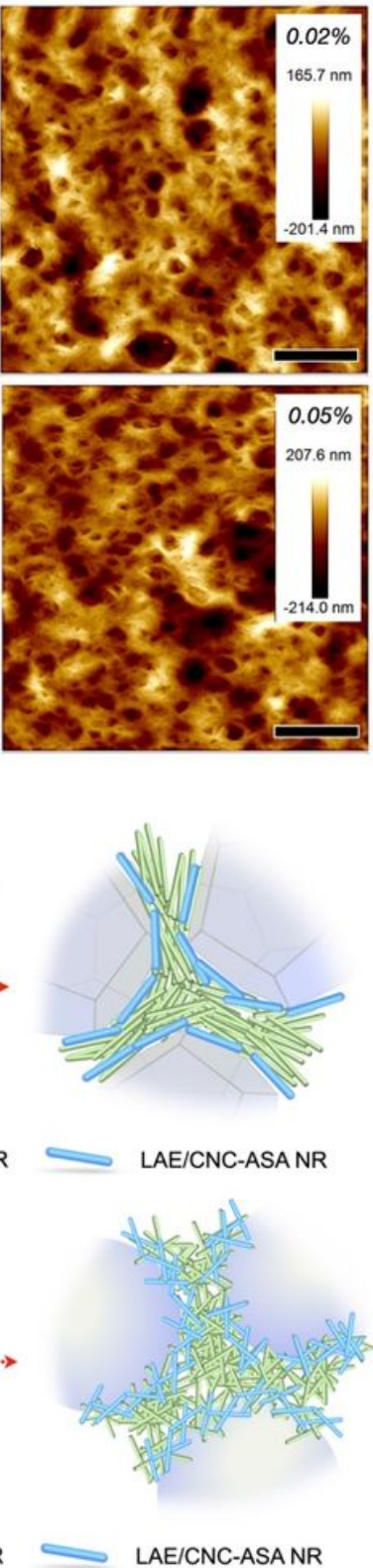

\section{Figure 5}

(a) Transmittance profiles of ASA Pickering emulsions with 2.0\% CNC and 0 0.05\% LAE (based on water phase) after 50 cycles of centrifugation at $2000 \mathrm{rpm}$. The horizontal axis indicates the height position along the test tube. All the samples were kept undisturbed at ambient temperature. (b) AFM images of LAE/CNC NRs-stabilized ASA Pickering emulsions with different content of LAE. All the scale bars are 2 $\mu \mathrm{m}$. The dosage of CNC was fixed to $2.0 \%$ and the oil (ASA)/water fraction was 1:2. (c) SEM image of ASA emulsions stabilized by CNC alone. (d) TEM image of emulsion droplets in (c). (e) SEM image of 
ASA emulsions stabilized by LAE/CNC with $C_{L A E} \sim 0.02 \%$. (f) TEM image of ASA droplets stabilized by LAE/CNC. $(g, h)$ Schematics of the stabilization mechanism among LAE/CNC NRs with and without LAE at the ASA/water interface.
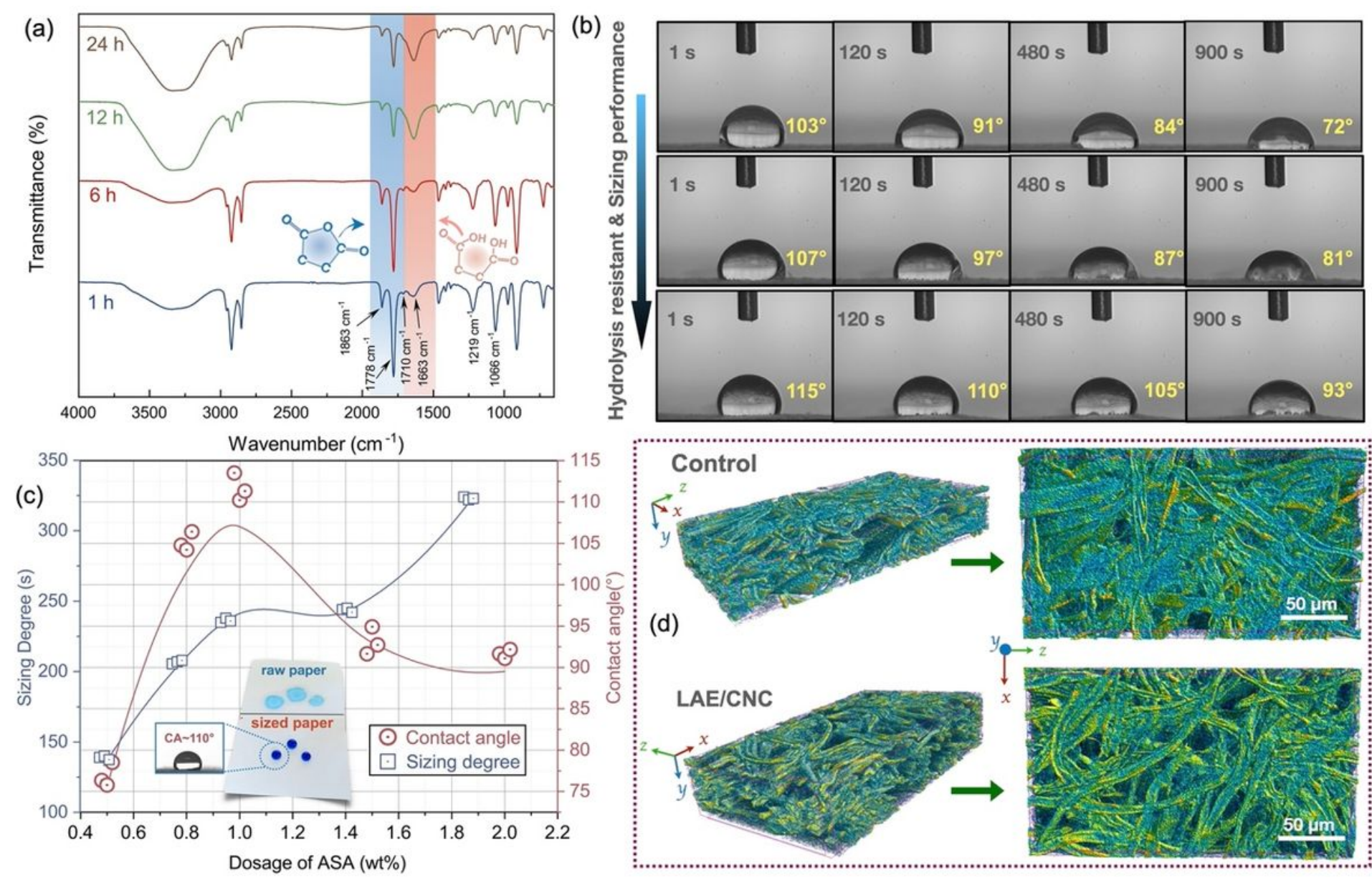

\section{Figure 6}

(a) FT-IR spectra of the ASA emulsion stabilized by LAE/CNC NRs at different storage time. (b) Optical images revealing the dynamic wetting behaviors of a water droplet $(\approx 25 \mu \mathrm{L})$ atop sized paper with 0.5 wt\% (top), $1.0 \mathrm{wt} \%$ (middle) and $2.0 \mathrm{wt} \%$ (bottom) CNC at room temperature. The LAE loading is fixed to 0.02 wt\%. (c) Sizing degree and contact angle of the sized paper by ASA based on LAE/CNC NRs with different ASA's dosage (wt\%, based on dry paper pulp (d) Top view of a micro-CT image of the sized paper with CNC-stabilized ASA and LAE/CNC-stabilized ASA, respectively. 


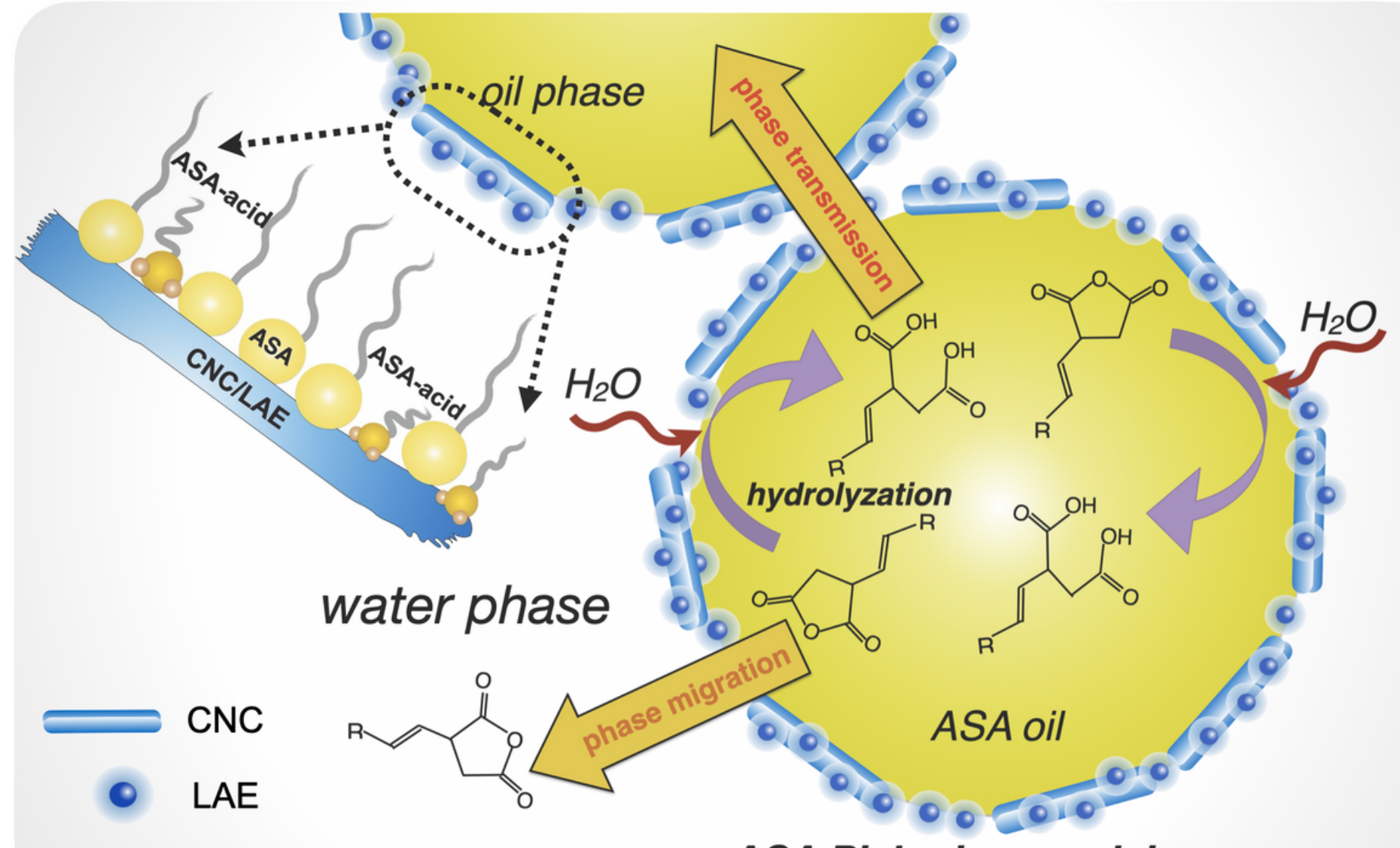

ASA Pickering emulsion

Figure 7

Conceptual schematic of the adsorption and chemical crosslinking of LAE/CNC NRs at ASA-water interfaces.

\section{Supplementary Files}

This is a list of supplementary files associated with this preprint. Click to download.

- SupportingInformation.docx

- Scheme1.png 